\title{
Comparison of Conventional and Modern Biomedical Tools Used for Diagnosis of Genital Tract Tuberculosis: An Overview
}

\author{
Gargi Kumari ${ }^{1,2}$, Divya Goel ${ }^{2}$ and Sanjay Mishra ${ }^{1, *}$ \\ ${ }^{I}$ School of Biotechnology, IFTM University, Lodhipur-Rajput, Delhi Road (NH 24), Moradabad 244 102, U.P., \\ India, ${ }^{2}$ Department of Clinical Biochemistry \&Pathology, Sri Sai Hospital, Delhi Road, Near Loco Shed Bridge, \\ Moradabad 244 001, U.P., India \\ *Corresponding Author: Email: sanjaymishra@iftmuniversity.ac.in
}

\begin{abstract}
Genital tuberculosis (TB) in women especially the Fallopian tubes and Endometrial is a major cause of chronic infertility in women in India. Due to the long and difficult treatment, it is mandatory to provide a definitive diagnosis of TB to help and motivate the patient for compliance. The present article is a compilation of comprehensive background literature concerning with comparison of conventional and modern biomedical tools used for diagnosis of genital tract $T B$, and may provide new insights into aiming to make a diagnosis in sterility patients that is due to $T B$, so that proper treatment can be given to treat the sterility.
\end{abstract}

Keywords: Genital tuberculosis; chronic infertility; Mycobacterium tuberculosis

\section{Introduction}

Mycobacterium tuberculosis has been identified as the aetiological agent of tuberculosis for many centuries. Genital tuberculosis is a chronic disease and often has low-grade symptomatology, with very few specific complaints [1-5]. Tuberculosis (TB) is a very common disease worldwide including India. Recent years have seen a resurgence of the disease and incidence is increasing day by day. Lungs are the most common organs involved but TB is a systemic illness and any organ of the body can be affected. Route of entry is through lungs. Contrary to common belief it does not spread by touch, close contact or sharing of utensils etc. The disease is caused by a type of bacteria called Mycobacterium tuberculosis. These bacteria spread by droplet infection. Patient with active disease release bacteria in air during coughing, sneezing, spitting etc. These bacteria remain suspended in air for long time. Thus the disease is more common in crowded, dark and unhygienic places. These bacteria enter the body through lungs. From here they can travel to any organ of the body and cause disease in that organ [3]. Female genital organs are one of the common sites to be involved in women. It is a chronic disease and bacteria way remain for long time slowly destroying the organs. The disease may remain totally symptomless or may lead to pelvic pain, fever, menstrual disturbances or vaginal discharge [4]. Sometimes fluid may accumulate in the abdomen. In very advance disease large pus filled masses may form in the tubes or ovaries. Infertility may be caused even by early or minimal disease. Fallopian tubes which transport eggs from the ovaries to the uterus are the most common organs involved and thus block the passages and lead to permanent infertility [4]. It is quite difficult to diagnose genital tuberculosis. The most important test is endometual biopsy i.e. testing the menstrual blood taken from the uterus. This can be obtained with the help of a thin instrument inserted in the uterus. It is a very simple procedure. The material thus obtained is sent to the laboratory for confirmation. This test is positive in only 50-60\% of cases. Other tests like X-ray chest, blood test, a skin test [4]. (M x test) is helpful but do not definitely prove or exclude the disease [5]. Ultrasound is another test which can give clues to the clinician. Endoscopic procedures i.e. laparoscopy and hysteroscopy also can pick up the signs of disease and also provide an opportunity to take samples for laboratory testing from various suspicious sites. Very effective medicines are now available, commonly called antitubercular therapy or ATT. The medicines are very effective in killing the bacteria. The treatment has to continue for a minimum of 6 months. In advanced cases a prolonged treatment may be required. However, the drugs cannot repair the damage done by TB. Fallopian tubes which are commonly damaged by the disease remain blocked and function cannot be easily restored even by surgery. All other symptoms like fever, pain, discharge etc. respond very well to the treatment. For large pus collection which does not respond to medicines, surgical treatment has to be done. Infertility is the commonest presentation. Almost 60 to $70 \%$ cases of genital TB present with infertility. In India almost $5-10 \%$ of all infertility is caused by genital TB. Medical treatment may restore fertility in early cases.

A study from South Africa found an incidence of $6 \%$ of culture-positive tuberculosis in an infertile population. The fallopian tubes are involved in most cases of genital tuberculosis and, together with endometrial involvement, cause infertility in patients. Many patients present with a symptom complex similar to that of ovarian carcinoma, i.e. abdominal distension, pelvic tumor and ascites, which may easily be confused with ovarian carcinoma. Besides, purposely it was projected to assess the outcome of in vitro fertilization and embryo transfer in tuberculosis infertility and to study the factors associated with success or failure of treatment. Ten patients with tuberculoses infertility underwent 22 cycles of in vitro fertilization and 9 cryopreserved-thawed 
embryo transfers at Bourn Hall Clinic. All patients underwent endometrial assessment by ultrasonography. Four patients had preliminary hysteroscopy, endometrial biopsy, and Doppler uterine blood flow studies. Six clinical pregnancies resulted in three live births in three patients and one current pregnancy in a fourth patient. There was one ectopic pregnancy and one twin pregnancy that aborted spontaneously at 14 weeks. The patients who had trophic endometrium achieved pregnancy at a rate of $42.9 \%$ (six of 14) (per embryo transfer) compared with $0 \%$ (none of. 14) 2 if the endometrium was atrophic. Conclusively, in vitro fertilization and embryo transfer offers the only realistic treatment for tuberculoses infertility. Preliminary assessment of the endometrium is helpful in assessing prognoses in these cases. Biopsies should be obtained by either laparoscopy or laparotomy if examination of the ascetic fluid could not confirm the diagnosis [6-10]. Although tuberculosis after the Second World War has been almost eradicated in the well developed countries due to vaccination, early diagnosis, proper antibiotics and increase in the life standards [11, 12], it still seems one of the major problems in the underdeveloped countries and also in the developing countries such as that of Turkey. Because immigration both from one country to another and also to cities, it seems that genital tuberculosis will reborn in many countries and areas where it is thought to be eradicated. Genital tuberculosis is generally secondary to a primary lesion within the body. In most cases primary lesions are pulmonary Genital tuberculosis is an elusive diagnosis and requires a high index of suspicion as a first step in the diagnostic process. Excellent cure rates are reported on all of the standard treatment regimens. Genital TB is always hard to diagnose [13, 14], because of the fact that it is a silent invader of the genital tract. The only reliable way of making a diagnosis is by actually culturing the tubercle bacillus from tissue sampled from the genital tract. Since it's nearly impossible to take tissue from the fallopian tubes, in practice this means that the diagnosis is usually made by finding tubercle bacilli in the endometrial tissue, obtained by uterine curettage or menstrual blood, or pleural and rarely gastrointestinal or renal $[15,16]$. In the past, Mycobacterium tuberculosis and Mycobacterium bovis were the organisms responsible for genital tuberculosis but now atypical varieties of Mycobacterium are being isolated [17]. In contrast to the Mycobacterium bovis and Mycobacterium tuberculosis, the other species of tuberculosis are not transmitted from person to person but from the environment with the mechanisms which are not fully understood [17]. Furthermore the tuberculosis caused by these varieties had no typical tuberculosis symptoms. Consequently, there is technical deficiency in the diagnosis and failure in the physician's decision making. Genital tuberculosis is an important cause of infertility and the cases with genital tuberculosis present with abdominal and pelvic pain and underwent laparotomy for abdominal mass or tubo-ovarian abscess [18]. The diagnosis is generally done during the operation. In this study we planned to evaluate genital Tuberculosis retrospectively among the infertile patients and to emphasize the role of tuberculosis in infertility.

Tuberculosis is known to spread through the blood stream and major sites affected in the genital tract is fallopian tubes by the invasion of peritoneum than uterus, especially endometrium is affected. Tuberculosis (TB) is still rampant in India [11, 12]; and TB of the genital tract is the commonest cause of tubal infertility. TB causes infertility only when it infects the genital tract (Genital TB). Sometimes latent bacilli that are left over from infections that the body successfully fights off can get reactivated, and then spread through the blood stream. It is only when it lodges and infects the genital tract, that TB can cause infertility. In the man it causes tuberculousepididymo-orchitis, blocking the passage, as a result of which the man becomes azoospermic (no sperm enter the semen because the tract is blocked). In the woman, it causes tuberculousendomteritis (infection of the uterus) and salpingitis (infection of the tubes). This infection can often be silent, and may not cause any symptoms or signs at all! [11-14].

While TB damages the fallopian tubes irreparably, it also damages the endometrium. In most women, if the diagnosis is made quickly and the infection treated promptly, the uterus heals well, partly because the old uterine lining is shed every month in the menstrual period, and a new one (which is healthy) regenerates. However, in severe cases, the TB endometritis does not heal, and leads to scarring and severe fibrosis and adhesions. These patients usually have scanty menses - and in some of them, the periods may stop completely, because the uterine lining has been burnt out. They have severe Asherman's syndrome (intrauterine adhesions); and this can be diagnosed by doing a hysteroscopy. Unfortunately, there is no effective treatment for this, as endometrial tissue after TB can become very avascular, and the only option for these unfortunate women is either surrogacy or adoption [11, 12]. In summary, the diagnosis of TB of the genital tract remains notoriously difficult to make. Most patients are misdiagnosed as having TB when in fact they don't, and many are treated for no good rhyme or reason The actual incidence of genital tuberculosis cannot be determined accurately in any population, since some of patients are asymptomatic, symptoms of the patients are not typical and especially in the well developed countries it is thought to be eradicated and the disease is discovered incidentally [14]. Tuberculosis was diagnosed unexpectedly in our patients during the laparotomy performed for different indications. Although normally our contemporary first line approach is laparoscopy for infertile cases, because of the retrospective nature of the presented study all mentioned infertile cases were administered to laparotomy for their pelvic masses. Schaefer estimated that $5-10 \%$ of infertile females the world over have genital tuberculosis, although this varies from less than $1 \%$ in the USA to nearly $13 \%$ in India [14]. The reproductive 
period is thought to be the most risky time to be infected by genital tuberculosis. In recent studies it was noticed that the mean age rose. According to Sutherland the mean age was 28.2 years in a time period from 1970 to 1980. Falk's series in Sweden also gave similar results [13,14]. Contrary to these results in Saudi Arabia between 1979 and 1983 Chadtopadhyayet al. noticed the mean age to be 24.8 [18]. In the presented series the mean age was 27.8 .

As a consequence of efforts made in this thrust area of clinical research, the modern pathological tools/techniques could be developed, although still certain number of modifications are required and/or thought to be incorporated in the existing ones taking into consideration the types of nutraceuticals being consumed as well as mode of nutrition:

TB Elisa A60: Tuberculosis (TB) is a common infectious disease worldwide especially in developing countries. Diagnosis of TB is difficult and often needs paraclinical studies. Measuring immunoglobulins against A-60 antigen of TB microorganism has been reported as a useful technique [19, 20]. The aim of this pathological technique dependent case study was to evaluate the diagnostic value of serologic test (IgA, $\operatorname{IgM}, \operatorname{IgG})$ against A60 antigen in tuberculosis. For this study, 176 TB patients (124 patients with smear-positive pulmonary TB and 52 patients with extra pulmonary TB) and 283 healthy controls were enrolled. Then, all subjects were tested for $\operatorname{IgM}, \operatorname{IgA}$ and $\operatorname{IgG}$ against A-60 antigen using ELISA technique. Sensitivities of IgM, IgG and $\operatorname{IgA}$ test were $15 \%, 25 \%$ and $40 \%$, respectively while the following specificities were also reported $100 \%, 75 \%$ and $90 \%$, respectively. Positive predictive values (PPV) were 100\%, 57\% and 72\%, however, negative predictive values (NPV) were $65 \%, 72 \%$ and $70 \%$, respectively. Combination of immunoglobulins' results increased sensitivity, for which the most sensitivity was observed in combination of $\operatorname{IgG}$ and $\operatorname{IgA}$ results. Genital tuberculosis is the disease of fourth decade in well developed countries, but it is encountered almost a decade ago in developing countries like Turkey and Saudi Arabia. This happening was tried to be explained with early marriage [19]. In a study from Pakistan, the majority of cases were between 25-45 years. The most common presenting symptoms were infertility (42.5\%) and abdominal pain (42\%). Others included fever, ascites, irregular vaginal bleeding, oligomenorrhea, chest pain and pain in the flanks [20]. With the classical knowledge, we know that genital tuberculosis is always secondary to a primary lesion elsewhere in the body and is spread by the hematogenous route. In recent years, genital tuberculosis is caused by the typical tuberculosis bacilli other than Mycobacterium tuberculosis, bovis and hominis. The atypical forms of bacilli do not show typical tuberculosis symptoms and are spread by environmental factors which predispose the genital tuberculosis. Genital tuberculosis is generally higher in the rural areas where the hygienic and economical status is lower, and vaccination cannot completely be performed. Conclusively, data obtained from this case study revealed the usefulness of serologic testing in TB diagnosis. Although its sensitivity is not high enough, combination of immunoglobulin results may improve the sensitivity $[19,20]$.

Antigen 60 IgG measurement: The identification of acid-fast bacilli (AFB) in sputum or tissue is the definite diagnosis of tuberculosis. However, this method of diagnosis is restricted by certain limitations. The serologic diagnosis of tuberculosis has been employed as one of the potent pathological technique for a long time [21]. The aim of this study was to determine the sensitivity and specificity of Antigen 60 (A60) immunoglobulin $G$ $(\mathrm{IgG})$ in patients with abnormal chest radiography and to assess its application in the serologic diagnosis of pulmonary tuberculosis. Data on patients who had been diagnosed using results of culture and pathology as having active pulmonary tuberculosis $(\mathrm{N}=178)$, other non-tuberculosis pulmonary disease $(\mathrm{N}=34)$, or no pulmonary disease $(\mathrm{N}=117)$ was collected from January 2001 through December 2002. The data of A60 IgG using enzyme-linked immunosorbent assay (ELISA), chest radiography, tuberculosis culture and pathology were obtained retrospectively. The cutoff value of A60 IgG was chosen according to a receiver operating characteristic (ROC) analysis. The sensitivity, specificity, positive predictive value, negative predictive value and likelihood ratio for positive and negative test were determined. The chosen cutoff value of 261.2 units defined the sensitivity (49.4\%) and specificity (79.5\%) of the test. The positive predictive value and likelihood ratio were $95.7 \%$ and 4.20 , respectively, for patients with abnormal chest radiography and $88.2 \%$ and 2.97 , respectively, for patients with abnormal chest radiography and negative AFB in sputum smear. Because of the high positive predictive value and likelihood ratio, a positive A60 IgG test in the presence of abnormal chest radiography can help make an accurate clinical diagnosis of pulmonary tuberculosis [21].

TB GOLD: Interferon-gamma release assays (IGRAs) are diagnostic tools for latent tuberculosis infection (LTBI). They are surrogate markers of Mycobacterium tuberculosis infection and indicate a cellular immune response to $M$. tuberculosis. IGRAs cannot distinguish between latent infection and active tuberculosis (TB) disease, and should not be used for diagnosis of active TB, which is a microbiological diagnosis. A positive IGRA result may not necessarily indicate active TB, and a negative IGRA result may not rule out active TB. Because IGRAs are not affected by BacilleCalmette-Guérin (BCG) vaccination status, IGRAs are useful for 
evaluation of LTBI in BCG-vaccinated individuals, particularly in settings where BCG vaccination is administered after infancy or multiple (booster) BCG vaccinations are given. In contrast, the specificity of tuberculin skin test (TST) varies depending on timing of BCG and whether repeated (booster) vaccinations are given. QuantiFERON, also known as QFT, is the registered trademark of the test for tuberculosis infection or latent tuberculosis. QFT is an interferon- $\gamma$ release assay (IGRA) used in tuberculosis diagnosis. The QFT-GIT assay is an ELISA-based, whole-blood test that uses peptides from three TB antigens (ESAT-6, CFP-10, and TB7.7) in an in-tube format. The result is reported as quantification of IFN-gamma in international units (IU) per $\mathrm{mL}$. An individual is considered positive for M. tuberculosis infection if the IFN-gamma response to TB antigens is above the test cut-off (after subtracting the background IFN-gamma response in the negative control).

QuantiFERON-TB Gold QFT-G and QuantiFERON-TB Gold In-Tube QFT-GIT, have replaced QuantiFERON-TB (QFT), which is no longer marketed. According to the U.S. Centers for Disease Control, [22] in 2001, the QuantiFERON-TB test (QFT) was approved by the Food and Drug Administration (FDA) as an aid for detecting latent Mycobacterium tuberculosis infection. This test is an in vitro diagnostic aid that measures a component of cell-mediated immune reactivity to $M$. tuberculosis. The test is based on the quantification of interferon-gamma (IFN- $\gamma$ ) released from sensitized lymphocytes in whole blood incubated overnight with purified protein derivative (PPD) from M. tuberculosis and control antigens. Tuberculin skin testing (TST) has been used for years as an aid in diagnosing latent tuberculosis infection (LTBI) and includes measurement of the delayed type hypersensitivity response 48-72 hours after intradermal injection of PPD. TST and QFT do not measure the same components of the immunologic response and are not interchangeable. Assessment of the accuracy of these tests is limited by lack of a standard for confirming LTBI. Compared with TST, QFT results are less subject to reader bias and error. In a CDC-sponsored multicenter trial, QFT and TST results were moderately concordant (overall kappa value $=0.60$ ). The level of concordance was adversely affected by prior bacilleCalmette-Guérin (BCG) vaccination, immune reactivity to nontuberculous mycobacteria (NTM), and a prior positive TST [23]. In addition to the multicenter study, two other published studies have demonstrated moderate concordance between TST and QFT. [24, 25]. However, one of the five sites involved in the CDC study reported less agreement [26]. Although there have been studies confirming the increased future risk of active TB in individuals with positive TST, the same was not true for those with a positive IGRA result. A recently published study [26] demonstrated that a positive IGRA result is predictive of future active TB risk. Moreover, IGRA was at least as sensitive and was more specific compared to traditional TST. In this study of immunocompetent recently exposed close contacts of active TB cases, the progression rate to active disease among untreated QFT positive individuals was significantly greater than for untreated TST positives (14.6\% versus $2.3 \%$ ). Although the numbers were small, all of the close contacts who went on to develop active TB were QFT positive, but only $83 \%$ were TST positive [26].

As noted above, prior BCG vaccination can produce false positive TST results. In a study of military personnel returning from missions, about one-half of the positive TSTs were falsely positive [27]. In a more recent study of military returning from missions, Franken et al. [28] reported evidence suggesting false positive TST results are common and that QFT testing could guide more targeted treatment and alleviate unnecessary anti-tuberculous treatment. Limitations of QFT include the need to draw blood and process it within 12 hours after collection and limited laboratory and clinical experience with the assay. There is need for further study of the utility of QFT in predicting the progression to active tuberculosis, particularly in children and immunocompromised hosts.

According to the U.S. Centers for Disease Control [29], the QuantiFERON-TB Gold test (QFT-G) is a whole-blood test for use as an aid in diagnosing Mycobacterium tuberculosis infection, including latent tuberculosis infection (LTBI) and tuberculosis (TB) disease. This test was approved by the U.S. Food and Drug Administration (FDA) in 2005. Blood samples are mixed with antigens (substances that can produce an immune response) and controls. For QFT-G, the antigens include mixtures of synthetic peptides representing two M. tuberculosis proteins, ESAT-6 and CFP-10. After incubation of the blood with antigens for 16 to 24 hours, the amount of interferon-gamma (IFN-gamma) is measured. If the patient is infected with M. tuberculosis, their white blood cells will release IFN-gamma in response to contact with the TB antigens. The QFT-G results are based on the amount of IFN-gamma that is released in response to the antigens. Clinical evaluation and additional tests (such as a chest radiograph, sputum smear, and culture) are needed to differentiate between a diagnosis of latent TB or active TB. Advantages of the test are: Requires a single patient visit to draw a blood sample. Results can be available within 24 hours; Does not boost responses measured by subsequent tests, which can happen with tuberculin skin tests (TST); Is not subject to reader bias that can occur with TST; Is not affected by prior BCG (bacilleCalmette-Guérin) vaccination. Besides, disadvantages and limitations of the test are: Blood samples must be processed within 12 hours after collection while white blood cells are still viable.

There are limited data on the use of QFT-G in children younger than 17 years of age, among persons recently exposed to $\mathrm{M}$. tuberculosis, and in immunocompromised persons (e.g., impaired immune function 
caused by HIV infection or acquired immunodeficiency syndrome [AIDS], current treatment with immunosuppressive drugs, selected hematological disorders, specific malignancies, diabetes, silicosis, and chronic renal failure). Errors in collecting or transporting blood specimens or in running and interpreting the assay can decrease the accuracy of QFT-G. Limited data on the use of QFT-G to determine who is at risk for developing TB disease. False positive results can occur with Mycobacterium szulgai, Mycobacterium kansasii, and Mycobacterium marinum [29].

PCR: Genital tuberculosis (GTB) is one of the major causes for severe tubal disease leading to infertility. Unlike pulmonary tuberculosis, the clinical diagnosis of GTB is difficult because in majority of cases the disease is either asymptomatic or has varied clinical presentation. Routine laboratory values are of little value in the diagnosis. An absolute diagnosis cannot be made from characteristic features in hysterosalpingogram (HSG) or laparoscopy. Due to the paucibacillary nature of GTB, diagnosis by mycobacterial culture and histopathological examination (HPE) have limitations and low detection rate. The objective of this case study [30] was to evaluate the efficacy of PCR technique, culture and histopathological examination in the diagnosis of GTB in female infertility. This study included 72 infertile women who met the inclusion and exclusion criteria. After a detailed history and clinical examination all patients were subjected to investigations including pelvic sonogram, HSG and laparoscopy. Endometrial samples from were allocated for AFB smear, culture and HPE examination. Only 49 samples were available for PCR using IS 6110 and TRC4 primers. In seven patients peritoneal fluid was also taken for culture and PCR. Based on the clinical profile and laparoscopic findings, a diagnostic criterion was derived to suspect GTB. Specific diagnostic tests were evaluated against this diagnostic criterion. Laparoscopy was suggestive of tuberculosis in 59.7 per cent of cases, AFB smear was positive in 8.3 per cent, culture was positive in 5.6 per cent, HPE positive in 6.9 per cent and PCR was positive in 36.7 per cent of cases. Based on the diagnostic criteria, GTB was suspected in 28 of the 49 cases. On evaluating against the diagnostic criteria, the sensitivity of PCR, HPE and culture were 57.1, 10.7, 7.14 per cent respectively. The concordance of results between the clinical criteria and specific diagnostic tests were analyzed by Kappa measure of agreement. The culture and HPE showed mild agreement with the clinical criteria, whereas PCR showed a moderate agreement. PCR was positive in two of the 21 cases in whom GTB was not suspected. False positive PCR in these two cases were ruled out by multiple areas of sampling and re-sampling in one case. The PCR results were negative in 12 of the 28 cases. PCR using TRC4 primers had a higher sensitivity (46.4\%) than IS 6110 primers (25\%) in detecting clinically suspected GTB. As shown previously, among the pulmonary tuberculosis patients $12.3 \%$ were diagnosed as genital tuberculosis and infertility was present in $71.4 \%$ of these patients [31]. With retrospective evaluation of the presented cases, we believe that each infertile patient, especially the ones from the poor socioeconomic population and immigrants should be thoroughly evaluated for tuberculosis. It should be noticed that all the diagnostic methods other than biopsy have high percentage of false negative findings. Therefore, laparoscopic or laparotomic biopsies must not be neglected if it a clinical suspicion exists. According to the experience collected from our own series, there isn't any clear cut diagnostic value of the methods other than biopsy. Recently, the PCR method is known to have an important diagnostic value. Conclusively the data obtained from this PCR based investigation reflected that conventional methods of diagnosis namely, HPE, AFB smear and culture have low sensitivity. PCR was found to be useful in diagnosing early disease as well as confirming diagnosis in clinically suspected cases. False negative PCR was an important limitation in this study [30].

\section{References}

[1] Botha, M.H. and Van der Merwe, F.H. (2008). Female genital tuberculosis. SA FamPract50 (5): $12-16$

[2] Angus, B.J., Yates, M., Conlon, C. and Byren, I. (2001). Cutaneous tuberculosis of the penis and sexual transmission of tuberculosis confirmed by molecular typing.Clinical Infectious Diseases33:132-4.

[3] Hassoun, A., Jacquette, G., Huang, A., Anderson, A., Smith, M.A.(2005). Female genital tuberculosis: uncommon presentation of tuberculosis in the United States. Am J Med118(11): 1295-9.

[4] Margolis, K., Wranz, P.A.B., Kruger, T.F., Joubert, J.J. and Odendaal, H.J. (1992). Genital tuberculosis at Tygerberg Hospital prevalence, clinical presentation and diagnosis. SAfr Med J 81:12-15.

[5] Marcus, S.F., Rizk, B., Fountain, S., Brinsden, P. (1994). Tuberculous infertility and in vitro fertilization. Am J ObstetGynecol171(6): 1593-6.

[6] Nogales-Ortiz, F., Tarancon, I. and Nogales, F.F. Jr. (1979). The pathology of female genital tuberculosis.A $31-y e a r$ study of 1436 cases.ObstetGynecol 53(4): 422-8.

[7] Sutherland, A.M. (1980). Surgical treatment of tuberculosis of the female genital tract.Br J ObstetGynaecol 87(7): 610-12.

[8] Saracoglu, O.F., Mungan, T. and Tanzer, F. (1992). Pelvic tuberculosis.Int J GynaecolObstet 37(2): 115-20.

[9] Raut, V.S., Mahashur, A.A. and Sheth, S.S. (2001). The Mantoux test in the diagnosis of genital tuberculosis in women.International Journal of Gynecology \& Obstetrics 72: 165-9.

[10] De Vynck, W.E., Kruger, T.F., Joubert, J.J., et al. (1990). Genital tuberculosis associated with female infertility in the Western Cape. S Afr Med J 77: 630-31.

[11] Dwivedi, N., Dwivedi, S.P., Kumar, A., Katoch, V.M. and Mishra, S. (2008). Computational identification of composite regulatory sites i8n 16s-rRNA gene promoters of Mycobacterium species. Bioinformation 2 (8): 363-8. 
[12] Mishra, S., Singh, R.B., Dwivedi, S.P., De Meester, F., Rybar, R., Pella, D., Fedacko, J. and Juneja, L.R. (2009). Effects of nutraceuticals on genetic expressions. The Open Nutraceuticals Journal 2:70-80.

[13] Coutour L., Delecour M., Leroy J.L. and Puech F. (1984). LaTuberculosegenitalexiste- telleencore?JGynecolObstetBiolReprod13:419-23.

[14] Jones H.W., Wentz A.C., Burnett LS. (1988). Novak's Textbook of Gynecology 11 $^{\text {th }}$ Ed. Baltimore, pp.557-70.

[15] Tourris H., Henrion R. and Delecour M. (1984). AbregeillustreGynecologieetObstetrique, pp. 201-5.

[16] Sfar E., Ouarda C. and Kharouf M. (1990). La Tuberculosegenitale feminine en Tunisie, Revue francaise de gynecologieetd'ostetrique 85 (6):359-63.

[17] Akinoglu A., Bilgin I. (1988). Tuberculous Enteritis and Peritonitis. The

Canadian Journal of Surgery 31(1):55-8.

[18] NamavarJahromi B., Parsanezhad M.E., Ghane-Shirazi R. (2001). Female genital tuberculosis and infertility.Int J GynaecolObstet75(3):269-72.

[19] Mahajan, M., Singh, N.P., Gadre, D.J., Talwar, V., Gupta, H.C., Agarwal, D.S. (1996).Detection of IgM antibodies in pulmonary tuberculosis by ELISA using A60 antigen.J Commun Disease 28 (3): 176-80.

[20] Ghadiri, K., Izadi, B., Afsharian, M., Vaziri, S., Rezaei, M. and Namdari, S. (2008). Diagnostic value of serological tests (IgA, IgG, IgM) against A-60 antigen in tuberculosis.Iranian Journal of Clinical Infectious Diseases 3(4): 205-8.

[21] Huang-Pin, W., Wen-Bin S., Wen-Bin, S., Fang-KeuiHsien, AHN, and Chung-Ching, H. (2004). The significance of Mycobacterium tuberculosis Antibody, Antigen $60 \mathrm{IgG}$ in patients with abnormal chest Radiography.Chang Gung Medical Journal 27 (12): 869-76.

[22] Gerald, H., Mazurek, M.D., Margarita, E., and Villarino, M.D. (2007). Guidelines for Using the QuantiFERON-TB Test for Diagnosing Latent Mycobacterium tuberculosis Infection. Retrieved 2007-06-01.

[23] Mazurek G.H., LoBue, P.A., Daley, C.L., et al. (2001). Comparison of a Whole-Blood Interferon Gamma Assay with Tuberculin Skin Testing for Detecting Latent Mycobacterium tuberculosis Infection.JAMA286:1740-47.

[24] Pottumarthy, S., Morris, A.J., Harrison, A.C. and Wells, V.C. (1999). Evaluation of the Tuberculin Gamma Interferon Assay: Potential to Replace the Mantoux Skin Test. J ClinMicrobiol37:3229-32.

[25] Streeton, J.A., Desem, N. and Jones, S.L. (1998). Sensitivity and Specificity of a Gamma Interferon Blood Test for Tuberculosis Infection.Int J Tuberc Lung Dis2:443-50.

[26] Bellete, B., Coberly, J., Barnes, G.L., et al. (2002). Evaluation of a Whole-Blood Interferon-Gamma Release Assay for the Detection of Mycobacterium tuberculosis Infection in 2 Study Populations. Clin Infect Dis34:1449-56.

[27] Diel, R., Loddenkemper, R., Meywald-Walter, K., Niemann, S. and Nienhaus, A. (2008). Predictive Value of a Whole Blood IFN- $\gamma$ Assay for the Development of Active Tuberculosis Disease after Recent Infection with Mycobacterium tuberculosis. Am $\mathbf{J}$ RespirCrit Care Med177:1164-70.

[28] Bruins, J., Gribnau, J.H. and Bwire, R. (1995). Investigation into Typical and Atypical Tuberculin Sensitivity in the Royal Netherlands Army, Resulting in a More Rational Indication for Isoniazid Prophylaxis. Tuber Lung Dis 76:540-44

[29] Franken, W.P.J., Timmermans, J.F., Prins, C., et al. (2007). Comparison of Mantoux and QuantiFERON TB Gold Tests for Diagnosis of Latent Tuberculosis Infection in Army Personnel.Clin Vaccine Immunol14:477-80.

[30] Aka N. and Vural E.Z. (1997). Evaluation of patients with active pulmonary tuberculosis for genital involvement. J ObstetGynaecol Res 23(4): 337-40. 